\title{
A Sharp Lieb-Thirring Inequality for Functional Difference Operators
}

Ari LAPTEV ${ }^{\mathrm{ab}}$ and Lukas SCHIMMER ${ }^{\mathrm{c}}$

a) Department of Mathematics, Imperial College London, London SW7 2AZ, UK

E-mail: a.laptev@imperial.ac.uk

b) Saint Petersburg State University, Saint Petersburg, Russia

c) Institut Mittag-Leffler, The Royal Swedish Academy of Sciences, 18260 Djursholm, Sweden E-mail: lukas.schimmer@kva.se

Received September 12, 2021, in final form November 25, 2021; Published online December 06, 2021 https://doi.org/10.3842/SIGMA.2021.105

\begin{abstract}
We prove sharp Lieb-Thirring type inequalities for the eigenvalues of a class of one-dimensional functional difference operators associated to mirror curves. We furthermore prove that the bottom of the essential spectrum of these operators is a resonance state.
\end{abstract}

Key words: Lieb-Thirring inequality; functional difference operator; semigroup property 2020 Mathematics Subject Classification: 47A75; 81Q10

To our friend and coauthor Leon Takhtajan on the occasion of his 70th birthday

\section{Introduction}

Let $P$ be the self-adjoint quantum mechanical momentum operator on $L^{2}(\mathbb{R})$, i.e., $P=-\mathrm{i} \frac{\mathrm{d}}{\mathrm{d} x}$ and for $b>0$ denote by $U(b)$ the Weyl operator $U(b)=\exp (-b P)$. By using the Fourier transform

$$
\widehat{\psi}(k)=(\mathcal{F} \psi)(k)=\int_{\mathbb{R}} \mathrm{e}^{-2 \pi \mathrm{i} k x} \psi(x) \mathrm{d} x
$$

we can write the domain of $U(b)$ as

$$
\operatorname{dom}(U(b))=\left\{\psi \in L^{2}(\mathbb{R}): \mathrm{e}^{-2 \pi b k} \widehat{\psi}(k) \in L^{2}(\mathbb{R})\right\} .
$$

Equivalently, $\operatorname{dom}(U(b))$ consists of those functions $\psi(x)$ which admit an analytic continuation to the strip $\{z=x+\mathrm{i} y \in \mathbb{C}: 0<y<b\}$ such that $\psi(x+\mathrm{i} y) \in L^{2}(\mathbb{R})$ for all $0 \leq y<b$ and there is a limit $\psi(x+\mathrm{i} b-\mathrm{i} 0)=\lim _{\varepsilon \rightarrow 0^{+}} \psi(x+\mathrm{i} b-\mathrm{i} \varepsilon)$ in the sense of convergence in $L^{2}(\mathbb{R})$, which we will denote simply by $\psi(x+\mathrm{i} b)$. The domain of the inverse operator $U(b)^{-1}$ can be characterised similarly.

For $b>0$ we define the operator $W_{0}(b)=U(b)+U(b)^{-1}=2 \cosh (b P)$ on the domain

$$
\operatorname{dom}\left(W_{0}(b)\right)=\left\{\psi \in L^{2}(\mathbb{R}): 2 \cosh (2 \pi b k) \widehat{\psi}(k) \in L^{2}(\mathbb{R})\right\} .
$$

The operator $W_{0}(b)$ is self-adjoint and unitarily equivalent to the multiplication operator $2 \cosh (2 \pi b k)$ in Fourier space. Its spectrum is thus absolutely continuous covering the interval $[2, \infty)$ doubly.

This paper is a contribution to the Special Issue on Mathematics of Integrable Systems: Classical and Quantum in honor of Leon Takhtajan.

The full collection is available at https://www.emis.de/journals/SIGMA/Takhtajan.html 
Let $V \geq 0, V \in L^{1}(\mathbb{R})$ now be a real-valued potential function. The scalar inequality $2 \cosh (2 \pi b k)-2 \geq(2 \pi b k)^{2}$ implies the operator inequality

$$
W_{0}(b)-2 \geq-b^{2} \frac{\mathrm{d}^{2}}{\mathrm{~d} x^{2}}
$$

on $\operatorname{dom}\left(W_{0}(b)\right)$. By Sobolev's inequality, we can conclude that the operator

$$
W_{V}(b)=W_{0}(b)-V
$$

is symmetric and bounded from below on the common domain of $W_{0}(b)$ and $V$. We can thus consider its Friedrichs extension, which we continue to denote by $W_{V}(b)$. This operator acts as

$$
\left(W_{V}(b) \psi\right)(x)=\psi(x+\mathrm{i} b)+\psi(x-\mathrm{i} b)-V(x) \psi(x) .
$$

Furthermore, by an application of Weyl's theorem (in a version for quadratic forms) and Rellich's lemma together with the fact that the form domain of $W_{0}(b)$ is continuously embedded in $H^{1}(\mathbb{R})$ (as discussed at the beginning of Section 4 ) the spectrum of $W_{V}(b)$ consists of essential spectrum $[2, \infty)$ and discrete finite-multiplicity eigenvalues below. Details of this argument in the similar case of a Schrödinger operator can be found in the upcoming book [2, Proposition 4.14].

We will show that the discrete spectrum satisfies a version of Lieb-Thirring inequalities for $1 / 2$-Riesz means. When formulating the main result of the paper it is convenient to parametrise the eigenvalues (repeated with multiplicities) as $\lambda_{j}=-2 \cos \left(\omega_{j}\right)$, where $\omega_{j} \in[0, \pi]$ for $\lambda_{j} \in$ $[-2,2]$ and $\omega_{j} \in \mathrm{i}[0, \infty)$ for $\lambda_{j} \leq-2$. Note that in the latter case $\lambda_{j}=-2 \cosh \left(\left|\omega_{j}\right|\right)$.

Theorem 1.1. Let $V \geq 0$ and let $V \in L^{1}(\mathbb{R})$. If $W_{V}(b) \geq-2$, then the discrete eigenvalues $\lambda_{j}=-2 \cos \left(\omega_{j}\right) \in[-2,2)$ (repeated with multiplicities) satisfy

$$
\sum_{j \geq 1} \frac{\sin \omega_{j}}{\omega_{j}} \leq \frac{1}{2 \pi b} \int_{\mathbb{R}} V(x) \mathrm{d} x .
$$

The constant in the inequality (1.2) is sharp in the sense that there is a potential $V$ such that (1.2) becomes equality.

Remark 1.2. Note that Theorem 1.1 does not allow to estimate eigenvalues below -2 . In fact, from the proof of this theorem, the case of one eigenvalue below -2 could be included in the inequality (1.2). We expect that the inequality holds true for all eigenvalues below -2 . However, the method we use in the proof prevents us from including all eigenvalues due to oscillating properties of the resolvent $\left(W_{0}(b)-\lambda\right)^{-1}$ for $\lambda<-2$.

Lieb-Thirring inequalities were first established for Schrödinger operators in [15]. For a onedimensional Schrödinger operator $-\frac{\mathrm{d}}{\mathrm{d} x^{2}}-V$ on $L^{2}(\mathbb{R})$ with negative eigenvalues $\mu_{1} \leq \mu_{2} \leq \cdots<0$, these bounds state that for any $\gamma \geq 1 / 2$ there is a constant $L_{\gamma}>0$ such that

$$
\sum_{j \geq 1}\left|\mu_{j}\right|^{\gamma} \leq L_{\gamma} \int_{\mathbb{R}} V(x)^{\gamma+1 / 2} \mathrm{~d} x
$$

for all $V \geq 0, V \in L^{\gamma+1 / 2}(\mathbb{R})$. The condition $\gamma \geq 1 / 2$ is optimal. Inequality (1.1) implies that

$$
\sum_{j \geq 1}\left|\lambda_{j}-2\right|^{\gamma} \leq \frac{L_{\gamma}}{b} \int_{\mathbb{R}} V(x)^{\gamma+1 / 2} \mathrm{~d} x
$$

for all eigenvalues $\lambda_{j} \leq 2$ of $W_{V}(b)$. Under the additional assumption $W_{V}(b) \geq-2$, our bound (1.2) presents an improvement of (1.4) for $\gamma=1 / 2$. This can be seen from the fact 
that for $\gamma=1 / 2$ the sharp constant in (1.3) is given by $L_{1 / 2}=1 / 2$ [7] and from the strict inequality

$$
\left|\lambda_{j}-2\right|^{\frac{1}{2}}=\left|2 \cos \omega_{j}+2\right|^{\frac{1}{2}}<\frac{\pi \sin \omega_{j}}{\omega_{j}}
$$

for $\omega_{j} \in[0, \pi)$. The difference of the terms above vanishes as $\omega_{j} \rightarrow \pi$, implying that (1.4) is asymptotically optimal for small coupling. While the necessity of $\gamma \geq 1 / 2$ in the Lieb-Thirring inequality for Schrödinger operators does not allow us to conclude that (1.4) fails for $0 \leq \gamma<1 / 2$, we will prove the following.

Theorem 1.3. Let $b>0$. If $V \in L^{1}(\mathbb{R})$ with $\int_{\mathbb{R}} V \mathrm{~d} x>0$, then $W_{V}(b)$ has at least one eigenvalue below 2. Furthermore, if $0 \leq \gamma<1 / 2$, then there is no constant $L_{\gamma}$ such that (1.4) holds for all compactly supported $V$. This conclusion holds even under the assumption that $W_{V}(b) \geq-2$.

The study of different properties of functional difference operators $W_{V}(b)$ was considered before. In the case when $-V=V_{0}=\mathrm{e}^{2 \pi b x}$ is an exponential function, the operator $W_{V_{0}}(b)$ first appeared in the study of the quantum Liouville model on the lattice [1] and plays an important role in the representation theory of the non-compact quantum group $\mathrm{SL}_{q}(2, \mathbb{R})$. The spectral analysis of this operator was first studied in [9], see also [17]. In the case when $-V=2 \cosh (2 \pi b x)$ the spectrum of $W_{V}(b)$ is discrete and converges to $+\infty$. Its Weyl asymptotics were obtained in [13]. This result was extended to a class of growing potentials in [14]. More information on spectral properties of functional difference operators can be found in papers $[4,5,10,11,16]$.

The proof method of Theorem 1.1 is similar to the proof of the sharp Lieb-Thirring inequality (1.3) for a one-dimensional Schrödinger operator in the case $\gamma=1 / 2$ as presented in [6]. It relies on a property of convolutions of the resolvent kernels of the operator under consideration. Such a semigroup property was also recently established for Jacobi operators where it was again used to prove sharp Lieb-Thirring type inequalities [12]. With a different proof (not using the convolution property) the sharp inequalities for the Schrödinger operator and the Jacobi operator were first obtained in [7] and in [8], respectively. Despite formal similarity to the case of Jacobi operators, it is still surprising that the proof method works for functional difference operators $W_{V}(b)$. These operators could be considered as differential operators of infinite order since the symbol $\cosh (2 \pi b k)$ can be written as an infinite Taylor series of symbols of even degree w.r.t. the variable $k$.

\section{Free resolvent}

Since $W_{0}(b) \geq 2$ we conclude that $W_{0}(b)-\lambda$ is an invertible operator for $\lambda<2$. Let $\lambda=-2 \cos (\omega)$ with $\omega \in[0, \pi]$ if $\lambda \in[-2,2]$ and $\omega \in \mathrm{i}[0, \infty)$ if $\lambda<-2$. Then in Fourier space the inverse of $W_{0}(b)-\lambda$ is given by the multiplication operator $(2 \cosh (2 \pi b k)+2 \cos (\omega))^{-1}$.

Applying the inverse Fourier transform $\mathcal{F}^{-1}$ to $(2 \cosh (2 \pi b k)+2 \cos (\omega))^{-1}$ we find the kernel of the free resolvent $G_{\lambda}=\left(W_{0}(b)-\lambda\right)^{-1}$ that is

$$
G_{\lambda}(x, y)=G_{\lambda}(x-y)=\frac{1}{2 b \sin \omega} \frac{\sinh \left(\frac{\omega}{b}(x-y)\right)}{\sinh \left(\frac{\pi}{b}(x-y)\right)} .
$$

Remark 2.1. Note that $G_{\lambda}(x-y)$ is an even and positive kernel for $\omega \in[0, \pi]$ and it becomes oscillating if $\omega \in \mathrm{i}(0, \infty)$. This fact is one of the reasons why we are able to study Lieb-Thirring inequalities only for the eigenvalues $\lambda_{j} \in[-2,2]$. This interval contains all of the discrete spectrum if the potential $V$ is "small" enough. However, if $V$ generates eigenvalues lying in $(-\infty,-2)$, then the oscillating property of the Green's function prevents us from obtaining the desired inequality for all eigenvalues. 
Note that the value of $G_{\lambda}$ on the diagonal $x=y$ takes the form

$$
G_{\lambda}(0)=\frac{1}{2 \pi b} \frac{\omega}{\sin \omega}
$$

and we can see the relation between the right-hand side of (2.2) and the expression in the left-hand side of (1.2). Due to our parameterisation of the spectral parameter, the convergence $\lambda \rightarrow 2^{-}$implies $\omega \rightarrow \pi^{-}$and thus

$$
G_{\lambda}(0) \sim \frac{1}{2 b} \frac{1}{\sqrt{1-\cos ^{2} \omega}} \sim \frac{1}{2 b} \frac{1}{\sqrt{2-\lambda}} \quad \text { as } \quad \lambda \rightarrow 2^{-} .
$$

If $\lambda \rightarrow-\infty$, then $\omega \rightarrow \mathrm{i} \infty$ and

$$
G_{\lambda}(0) \sim \frac{1}{\pi b}|\lambda|^{-1} \log |\lambda| .
$$

In [17] L. Faddeev and L.A. Takhtajan studied the resolvent in a slightly different form

$$
G_{\lambda}(x, y)=\frac{\sigma}{\sinh \left(\frac{\pi \mathrm{i} \varkappa}{\sigma}\right)}\left(\frac{\mathrm{e}^{-2 \pi \mathrm{i} \varkappa(x-y)}}{1-\mathrm{e}^{-4 \pi \mathrm{i} \sigma(x-y)}}+\frac{\mathrm{e}^{2 \pi \mathrm{i} \varkappa(x-y)}}{1-\mathrm{e}^{4 \pi \mathrm{i} \sigma(x-y)}}\right),
$$

which coincides with (2.1) with $\sigma=\mathrm{i} / 2 b, \lambda=2 \cosh (2 b \pi \varkappa)$ and $\varkappa=\frac{\omega-\pi}{2 \pi \mathrm{i} b}$. It was pointed out that the free resolvent can be written using the analogues of the Jost solutions

$$
f_{-}(x, \varkappa)=\mathrm{e}^{-2 \pi \mathrm{i} \varkappa x} \quad \text { and } \quad f_{+}(x, \varkappa)=\mathrm{e}^{2 \pi \mathrm{i} \varkappa x}
$$

that appear in the theory of one-dimensional Schrödinger operators. Namely

$$
G_{\lambda}(x-y)=\frac{2 \sigma}{C\left(f_{-}, f_{+}\right)(\varkappa)}\left(\frac{f_{-}(x, \varkappa) f_{+}(y, \varkappa)}{1-\mathrm{e}^{\frac{\pi \mathrm{i}}{\sigma^{\prime}}(x-y)}}+\frac{f_{-}(y, \varkappa) f_{+}(x, \varkappa)}{1-\mathrm{e}^{-\frac{\pi \mathrm{i}}{\sigma^{\prime}}(x-y)}}\right),
$$

where $\sigma^{\prime} \sigma=-1 / 4$ and where $C(f, g)$ is the so-called Casorati determinant (a difference analogue of the Wronskian) of the solutions of the functional-difference equation

$$
C(f, g)(x, \varkappa)=f\left(x+2 \sigma^{\prime}, \varkappa\right) g(x, \varkappa)-f(x, \varkappa) g\left(x+2 \sigma^{\prime}, \varkappa\right) .
$$

For the Jost solutions $C\left(f_{-}, f_{+}\right)(x, \varkappa)=2 \sinh \left(\frac{\pi \mathrm{i} \kappa}{\sigma}\right)$.

The equality $\left(W_{0}(b)-\lambda\right) G(x-y)=\delta(x-y)$ could be interpreted as an equation of distributions. Since the functions $f_{ \pm}(x, k)$ are Jost solutions, the distribution defined by $\left(W_{0}(b)-\lambda\right) \times$ $G(x-y)$ is supported only at $x=y$, and its singular part coincides with the singular part of the distribution

$$
\begin{aligned}
-\frac{2 \sigma \sigma^{\prime}}{\pi \mathrm{i} C\left(f_{-}, f_{+}\right)(\varkappa)}( & \frac{f_{-}\left(x+2 \sigma^{\prime}, \varkappa\right) f_{+}(y, \varkappa)-f_{-}(y, \varkappa) f_{+}\left(x+2 \sigma^{\prime}, \varkappa\right)}{x-y-\mathrm{i} 0} \\
& \left.+\frac{f_{-}\left(x-2 \sigma^{\prime}, \varkappa\right) f_{+}(y, \varkappa)-f_{-}(y, \varkappa) f_{+}\left(x-2 \sigma^{\prime}, \varkappa\right)}{x-y+\mathrm{i} 0}\right)
\end{aligned}
$$

in the neighbourhood of $x=y$. This singular part is equal to

$$
-\frac{2 \sigma \sigma^{\prime}}{\pi \mathrm{i}}\left(\frac{1}{x-y-\mathrm{i} 0}-\frac{1}{x-y+\mathrm{i} 0}\right)=\delta(x-y)
$$

where the authors used the Sokhotski-Plemelj formula. This formula is similar to the respective formula for a Schrödinger operator when the Dirac $\delta$-function appears by differentiating a step function. 


\section{Proof of inequality (1.2)}

\subsection{Some auxiliary results}

We first collect some results from [6] verbatim. Let $A$ be a compact operator on a Hilbert space $\mathcal{G}$ and let us denote

$$
\|A\|_{n}=\sum_{j=1}^{n} \sqrt{\lambda_{j}\left(A^{*} A\right)},
$$

where $\lambda_{j}\left(A^{*} A\right)$ are the eigenvalues of $A^{*} A$ in decreasing order. Then by Ky Fan's inequality (see for example [3, Lemma 4.2]) the functionals $\|\cdot\|_{n}, n=1,2, \ldots$, are norms and thus for any unitary operator $Y$ in $\mathcal{G}$ we have

$$
\left\|Y^{*} A Y\right\|_{n}=\|A\|_{n}
$$

Definition 3.1. Let $A, B$ be two compact operators on $\mathcal{G}$. We say that $A$ majorises $B$ or $B \prec A$, iff

$$
\|B\|_{n} \leq\|A\|_{n}, \quad \text { for all } \quad n \in \mathbb{N} .
$$

Lemma 3.2. Let $A$ be a nonnegative compact operator acting in $\mathcal{G},\{Y(k)\}_{k \in \mathbb{R}}$ be a family of unitary operators on $\mathcal{G}$, and let $g(k) \mathrm{d} k$ be a probability measure on $\mathbb{R}$. Then the operator

$$
B=\int_{\mathbb{R}} Y(k)^{*} A Y(k) g(k) \mathrm{d} k
$$

is majorised by $A$.

Proof. This is a simple consequence of the triangle inequality

$$
\|B\|_{n} \leq \int_{\mathbb{R}}\left\|Y^{*}(k) A Y(k)\right\|_{n} g(k) \mathrm{d} k=\|A\|_{n} \int_{\mathbb{R}} g(k) \mathrm{d} k=\|A\|_{n} .
$$

Let $\lambda_{j}=-2 \cos \omega_{j} \leq 2$ be the eigenvalues of $W_{0}(b)-V$ with $V \geq 0$. In order to slightly simplify the notations it is convenient to write

$$
\lambda_{j}=-2 \cos \left(\sqrt{\theta_{j}}\right)
$$

with $\theta_{j} \in\left(-\infty, \pi^{2}\right]$ and $\omega_{j}^{2}=\theta_{j}$.

Let us denote by $K_{\lambda}$ the Birman-Schwinger operator

$$
K_{\lambda}=V^{1 / 2} G_{\lambda} V^{1 / 2}
$$

Let $\mu_{j}\left(K_{\lambda}\right)$ be the eigenvalues (in decreasing order) of the Birman-Schwinger operator $K_{\lambda}$ defined in (3.1). Then due to the Birman-Schwinger principle we have

$$
1=\mu_{j}\left(K_{\lambda_{j}}\right)
$$

Let us define the operator

$$
L_{\theta}:=\frac{1}{G_{-2 \cos \sqrt{\theta}}(0)} K_{-2 \cos \sqrt{\theta}}
$$


where $G_{-2 \cos \sqrt{\theta}}(0)=\frac{1}{2 \pi b} \frac{\sqrt{\theta}}{\sin \sqrt{\theta}}$ is given in (2.2). Then from (3.2) we obtain

$$
\sum_{j \geq 1} \frac{1}{G_{\lambda_{j}}(0)}=\sum_{j \geq 1} \frac{1}{G_{\lambda_{j}}(0)} \mu_{j}\left(K_{\lambda_{j}}\right)=\sum_{j \geq 1} \mu_{j}\left(L_{\theta_{j}}\right)
$$

The integral kernel of the operator $L_{\theta}$ is given by $\sqrt{V(x)} g_{\pi^{2}, \theta}(x-y) \sqrt{V(y)}$, where

$$
g_{\pi^{2}, \theta}(x):=\frac{\pi}{\sqrt{\theta}} \frac{\sinh \left(\frac{\sqrt{\theta}}{b} x\right)}{\sinh \left(\frac{\pi}{b} x\right)} .
$$

Consider a more general function

$$
g_{\varphi, \theta}(x):=\frac{\sqrt{\varphi}}{\sqrt{\theta}} \frac{\sinh \left(\frac{\sqrt{\theta}}{b} x\right)}{\sinh \left(\frac{\sqrt{\varphi}}{b} x\right)}
$$

Since $g_{\varphi, \theta}(0)=1$ its Fourier transform $\widehat{g}_{\varphi, \theta}=\mathcal{F}\left(g_{\varphi, \theta}\right)$ satisfies the equation

$$
\int_{\mathbb{R}} \widehat{g}_{\varphi, \theta}(k) \mathrm{d} k=1
$$

Moreover, for any $-\infty<\theta<\varphi$ with $0<\varphi<\pi^{2}$ we have

$$
\widehat{g}_{\varphi, \theta}(k)=\mathcal{F}\left(\frac{\sqrt{\varphi}}{\sqrt{\theta}} \frac{\sinh \left(\frac{\sqrt{\theta}}{b} x\right)}{\sinh \left(\frac{\sqrt{\varphi}}{b} x\right)}\right)(k)=\frac{2 \pi \sin \left(\pi \frac{\sqrt{\theta}}{\sqrt{\varphi}}\right)}{\sqrt{\theta}} \frac{b}{2 \cosh \left(\frac{2 \pi^{2} b k}{\sqrt{\varphi}}\right)+2 \cos \left(\frac{\pi \sqrt{\theta}}{\sqrt{\varphi}}\right)},
$$

and the right-hand side is positive. Thus $\widehat{g}_{\varphi, \theta} \mathrm{d} k$ is a probability measure for such values.

Note also that importantly

$$
\frac{g_{\pi^{2}, \theta}(x)}{g_{\pi^{2}, \theta^{\prime}}(x)}=\frac{\sqrt{\theta^{\prime}}}{\sqrt{\theta}} \frac{\sinh \left(\frac{\sqrt{\theta}}{b} x\right)}{\sinh \left(\frac{\sqrt{\theta^{\prime}}}{b} x\right)}=g_{\theta^{\prime}, \theta}(x)
$$

and therefore

$$
\left(\widehat{g}_{\pi^{2}, \theta^{\prime}} * \widehat{g}_{\theta^{\prime}, \theta}\right)(k)=\widehat{g}_{\pi^{2}, \theta}(k) .
$$

This is the interesting convolution/semigroup property mentioned in the introduction. In the special case $-\infty<\theta<0=\theta^{\prime}$ analogous computations lead to the same result with $\widehat{g}_{0, \theta}(k)=$ $\chi_{[-1,1]}(2 \pi b k / \sqrt{|\theta|}) \pi b / \sqrt{|\theta|}$.

Lemma 3.3 (monotonicity). For $\left(\theta, \theta^{\prime}\right)$ such that $-\infty<\theta \leq \theta^{\prime}$ and $0 \leq \theta^{\prime}<\pi^{2}$ we have $L_{\theta} \prec L_{\theta^{\prime}}$.

Proof. Let $Y(k): L^{2}(\mathbb{R}) \rightarrow L^{2}(\mathbb{R})$ be the unitary multiplication operator

$$
(Y(k) \psi)(x)=\mathrm{e}^{-2 \pi \mathrm{i} k x} \psi(x)
$$

and let $T$ be the projection onto $V^{1 / 2}$, i.e.,

$$
(T \psi)(x)=V^{1 / 2}(x) \int_{\mathbb{R}} V^{1 / 2}(y) \psi(y) \mathrm{d} y .
$$


Using $Y\left(k^{\prime}+k^{\prime \prime}\right)=Y\left(k^{\prime}\right) Y\left(k^{\prime \prime}\right)$ and Lemma 3.2 we obtain

$$
\begin{aligned}
L_{\theta} & =\int_{\mathbb{R}} Y(k)^{*} T Y(k) \widehat{g}_{\pi^{2}, \theta}(k) \mathrm{d} k \\
& =\int_{\mathbb{R}} \int_{\mathbb{R}} Y(k)^{*} T Y(k) \widehat{g}_{\pi^{2}, \theta^{\prime}}\left(k^{\prime}\right) \widehat{g}_{\theta^{\prime}, \theta}\left(k-k^{\prime}\right) \mathrm{d} k^{\prime} \mathrm{d} k \\
& =\int_{\mathbb{R}} Y\left(k^{\prime \prime}\right)^{*}\left(\int_{\mathbb{R}} Y\left(k^{\prime}\right)^{*} T Y\left(k^{\prime}\right) \widehat{g}_{\pi^{2}, \theta^{\prime}}\left(k^{\prime}\right) \mathrm{d} k^{\prime}\right) Y\left(k^{\prime \prime}\right) \widehat{g}_{\theta^{\prime}, \theta}\left(k^{\prime \prime}\right) \mathrm{d} k^{\prime \prime} \prec L_{\theta^{\prime}},
\end{aligned}
$$

where we have used that $\widehat{g}_{\theta^{\prime}, \theta} \mathrm{d} k$ is a probability measure.

Remark 3.4. With a slight abuse of notations, Lemma 3.3 says that $L_{\lambda} \prec L_{\lambda^{\prime}}$ for any $\lambda<2$ as long as $\lambda \leq \lambda^{\prime}$ and $-2 \leq \lambda^{\prime}<2$.

\subsection{Proof of inequality (1.2)}

We now enumerate the eigenvalues of the operator $W_{V}(b)$ belonging to the interval $[-2,2)$ such that $-2 \leq \lambda_{1} \leq \lambda_{2} \leq \lambda_{3} \leq \cdots$ repeated with multiplicity. By using the monotonicity established in Lemma 3.3 we have a sequence of inequalities

$$
\begin{aligned}
& \frac{1}{G_{\lambda_{1}}(0)}=2 \pi b \frac{\sin \omega_{1}}{\omega_{1}}=\mu_{1}\left(L_{\theta_{1}}\right) \leq \mu_{1}\left(L_{\theta_{2}}\right), \\
& \sum_{j=1}^{2} \frac{1}{G_{\lambda_{j}}(0)}=2 \pi b \sum_{j=1}^{2} \frac{\sin \omega_{j}}{\omega_{j}} \leq \sum_{j=1}^{2} \mu_{j}\left(L_{\theta_{2}}\right) \leq \sum_{j=1}^{2} \mu_{j}\left(L_{\theta_{3}}\right), \\
& \sum_{j=1}^{3} \frac{1}{G_{\lambda_{j}}(0)}=2 \pi b \sum_{j=1}^{3} \frac{\sin \omega_{j}}{\omega_{j}} \leq \sum_{j=1}^{3} \mu_{j}\left(L_{\theta_{3}}\right) \leq \sum_{j=1}^{3} \mu_{j}\left(L_{\theta_{4}}\right), \quad \text { etc. }
\end{aligned}
$$

Note that we do not use any assumptions on the multiplicities of the eigenvalues, other than their finiteness. Furthermore, by Lemma 3.3 the same results also hold true if a single eigenvalue is below -2 . Continuing the above process and noting that the trace of $L_{\theta}$ is $\int_{\mathbb{R}} V \mathrm{~d} x$ for all $\theta$, we finally obtain

$$
\sum_{j \geq 1} \frac{\sin \omega_{j}}{\omega_{j}} \leq \frac{1}{2 \pi b} \int_{\mathbb{R}} V(x) \mathrm{d} x .
$$

The proof is complete.

Remark 3.5. Note that $\frac{2 \cosh (2 \pi b k)-2}{b^{2}} \rightarrow(2 \pi k)^{2}$ tends to the symbol of the second derivative as $b \rightarrow 0$ and that $W_{b^{2} V}(b) \geq-2$ for sufficiently small $b$. We thus expect that it should be possible to recover the Lieb-Thirring inequality (1.3) for a Schrödinger operator with the sharp constant $L_{1 / 2}=1 / 2$ from Theorem 1.1 .

\section{Sharpness of inequality (1.2)}

Similarly to the case of Schrödinger operators, we aim to prove that the Lieb-Thirring inequality becomes an equality for Dirac-delta potentials. To this end let $c>0$ and consider the potential $V_{c}(x)=c \delta(x)$. To properly define $W_{V_{c}}(b)$, we first note that the quadratic form $\left\langle\psi,\left(W_{0}(b)-2\right) \psi\right\rangle$ can be written as

$$
\left\langle\psi,\left(W_{0}(b)-2\right) \psi\right\rangle=\int_{\mathbb{R}}|2 \sinh (\pi b k) \widehat{\psi}(k)|^{2} \mathrm{~d} k=\int_{\mathbb{R}}|\psi(x+\mathrm{i} b / 2)-\psi(x-\mathrm{i} b / 2)|^{2} \mathrm{~d} x .
$$


This can be seen by introducing the self-adjoint operator $D(b)=U(b / 2)-U(b / 2)^{-1}=2 \sinh \left(\frac{b P}{2}\right)$ and checking that $D(b)^{2}=W_{0}(b)-2$ either directly or by means of the identity $\cosh (2 \pi b k)-1$ $=2 \sinh (\pi b k)^{2}$. The form domain of $W_{0}(b)$ is thus $\operatorname{dom}(D(b))=\operatorname{dom}\left(W_{0}(b / 2)\right) \subset H^{1}(\mathbb{R})$ and on this domain Sobolev's inequality yields that

$$
\begin{aligned}
|\psi(0)|^{2} & \leq \varepsilon \int_{\mathbb{R}}\left|\psi^{\prime}(x)\right|^{2} \mathrm{~d} x+\frac{1}{\varepsilon} \int_{\mathbb{R}}|\psi(x)|^{2} \mathrm{~d} x \\
& \leq \frac{\varepsilon}{b^{2}} \int_{\mathbb{R}}|2 \sinh (\pi b k) \widehat{\psi}(k)|^{2} \mathrm{~d} k+\frac{1}{\varepsilon} \int_{\mathbb{R}}|\psi(x)|^{2} \mathrm{~d} x
\end{aligned}
$$

for any choice of $\varepsilon>0$. The KLMN theorem thus allows us to define $W_{0}(b)-V_{c}$. As a rank one perturbation of the operator $W_{0}(b)$ the potential $V_{c}$ generates no more than one eigenvalue below the continuous spectrum $[2, \infty)$.

In Fourier space the eigenequation $\left(W_{0}(b)-c \delta\right) \psi_{c}=\lambda \psi_{c}$ becomes

$$
2 \cosh (2 \pi b k) \widehat{\psi_{c}}(k)-c \psi_{c}(0)=\lambda \widehat{\psi_{c}}(k)
$$

by means of the formal identity $\mathcal{F}\left(\delta \psi_{c}\right)=\psi_{c}(0)$. Writing again $\lambda=-2 \cos \omega$ we obtain

$$
\widehat{\psi_{c}}(k)=\frac{c \psi_{c}(0)}{2 \cosh (2 \pi b k)+2 \cos \omega}
$$

and therefore

$$
\psi_{c}(x)=c \psi_{c}(0) G_{-2 \cos \omega}(x)=\frac{c \psi_{c}(0)}{2 b \sin \omega} \frac{\sinh \left(\frac{\omega}{b} x\right)}{\sinh \left(\frac{\pi}{b} x\right)} .
$$

Of course we could have seen this immediately by using the equation for the Green's function

$$
\left(W_{0}(b)+2 \cos \omega\right) G_{-2} \cos \omega(x)=\delta(x) .
$$

Letting $x \rightarrow 0$ in (4.3) we find

$$
1=\frac{c}{2 b \sin \omega} \frac{\omega}{\pi}
$$

or equivalently

$$
\frac{\sin \omega}{\omega}=\frac{c}{2 \pi b}
$$

Since $\frac{\sin \sqrt{\theta}}{\sqrt{\theta}}$ is a monotone decreasing function of $\theta=\omega^{2} \in\left(-\infty, \pi^{2}\right]$ that takes all values in $[0, \infty)$, for any $c>0$ there is a unique solution $\omega_{c}$ to $(4.4)$ and vice versa. If $c /(2 \pi b)<1$ then $\omega_{c} \in(0, \pi)$ and otherwise $\omega_{c} \in \mathrm{i}[0, \infty)$. Since $\int V_{c} \mathrm{~d} x=c$, the identity (4.4) can be rewritten as

$$
\frac{\sin \omega}{\omega}=\frac{1}{2 \pi b} \int_{\mathbb{R}} V_{c}(x) \mathrm{d} x
$$

showing that the Lieb-Thirring inequality is satisfied for potentials $-c \delta$ with a single eigenvalue that can be placed anywhere in $(-\infty, 2)$ by choosing $c>0$ suitably.

Remark 4.1. If we choose the normalising constant $\psi(0)>0$ then the eigenfunction defined in (4.3)

$$
\psi_{c}(x)=\frac{c \psi(0)}{2 b \sin \omega_{c}} \frac{\sinh \left(\frac{\omega_{c}}{b} x\right)}{\sinh \left(\frac{\pi}{b} x\right)}
$$


is positive assuming that the coupling constant $c$ is small enough satisfying the inequality $c /(2 \pi b) \leq 1$ and thus $\omega_{c} \in[0, \pi)$. Note that if $c /(2 \pi b)=1$ then $\omega_{c}=0$ and

$$
\psi_{c}(x)=\frac{\pi \psi(0) x}{b \sinh \left(\frac{\pi}{b} x\right)}>0 .
$$

However, if the coupling constant $c>2 \pi b$ then $\omega_{c} \in \mathrm{i}(0, \infty)$ and hence

$$
\psi_{c}(x)=\frac{c \psi(0)}{2 b \sinh \left|\omega_{c}\right|} \frac{\sin \left(\frac{\left|\omega_{c}\right|}{b} x\right)}{\sinh \left(\frac{\pi}{b} x\right)}
$$

is an oscillating function and in particular has an infinite number of zeros. This contradicts a possible conjecture that the eigenfunction for the lowest eigenvalue is strictly positive.

Open problem. Assume that the discrete spectrum $\sigma_{\mathrm{d}}\left(W_{V}(b)\right)$ of the operator $W_{V}(b)$ satisfies the property $\sigma_{\mathrm{d}}\left(W_{V}(b)\right) \subset[-2,2)$. Is it true that the eigenfunction corresponding to the lowest eigenvalue could be chosen strictly positive?

\section{$5 \quad$ Necessity of $\gamma \geq 1 / 2$}

The following argument is similar to that presented in the upcoming book [2, Propositions 4.41 and 4.42] for the case of a Schrödinger operator. For $\varepsilon>0$ let $\psi_{\varepsilon}(x)=1 / \cosh (2 \varepsilon x / b)$. If $\varepsilon$ is sufficiently small, say $\varepsilon \leq \varepsilon_{0}$, then $\psi_{\varepsilon} \in \operatorname{dom}\left(W_{0}(b)\right)$. Using (4.1) we compute that

$$
\left\langle\psi_{\varepsilon},\left(W_{0}(b)-2\right) \psi_{\varepsilon}\right\rangle=\frac{b \sin ^{2} \varepsilon}{2 \varepsilon} \int_{\mathbb{R}}\left|\frac{2 \sinh x}{\cos ^{2} \varepsilon \cosh ^{2} x+\sin ^{2} \varepsilon \sinh ^{2} x}\right|^{2} \mathrm{~d} x \leq C b \varepsilon
$$

for a constant $C>0$ independent of $\varepsilon \leq \varepsilon_{0}$. For any potential $V \in L^{1}(\mathbb{R})$ it holds that $\left\langle\psi_{\varepsilon}, V \psi_{\varepsilon}\right\rangle \rightarrow \int_{\mathbb{R}} V \mathrm{~d} x$ as $\varepsilon \rightarrow 0$ by dominated convergence and thus for sufficiently small $\varepsilon$

$$
\left\langle\psi_{\varepsilon},\left(W_{V}(b)-2\right) \psi_{\varepsilon}\right\rangle<0 .
$$

By the min-max principle this proves the first part of Theorem 1.3.

For the second assertion of the theorem we choose more specifically the compactly supported potential $V(x)=c \chi_{[-1 / 2,1 / 2]}(x / b)$. By Sobolev's inequality $W_{V}(b) \geq-2$ for sufficiently small $c \leq c_{0}$ such that all the discrete eigenvalues of $W_{V}(b)$ are contained in $[-2,2)$. Furthermore $\left\|\psi_{\varepsilon}\right\|^{2}=b / \varepsilon$ and, since $\tanh x \geq x / 2$ for $0 \leq x \leq 1$,

$$
\left\langle\psi_{\varepsilon}, V \psi_{\varepsilon}\right\rangle=c b \int_{-1 / 2}^{1 / 2}|\cosh (2 \varepsilon x)|^{-2} \mathrm{~d} x=\frac{c b \tanh \varepsilon}{\varepsilon} \geq \frac{1}{2} c b
$$

for $\varepsilon \leq 1$. We now choose $\varepsilon=c \delta$. If $\delta \leq \min \left(\varepsilon_{0} / c_{0}, 1 / c_{0}\right)$ such that $\varepsilon \leq \min \left(\varepsilon_{0}, 1\right)$, then (5.1) and (5.2) both hold and

$$
\frac{\left\langle\psi_{\varepsilon},\left(W_{V}(b)-2\right) \psi_{\varepsilon}\right\rangle}{\left\|\psi_{\varepsilon}\right\|^{2}} \leq C \varepsilon^{2}-\frac{1}{2} c \varepsilon=c^{2} \delta\left(C \delta-\frac{1}{2}\right) .
$$

Choosing $\delta<\min \left(\varepsilon_{0} / c_{0}, 1 / c_{0}, 1 / 2 C\right)$ we can conclude by the min-max principle that $W_{V}(b)-2$ has a negative eigenvalue $\lambda_{1} \leq-c^{2} \delta\left(\frac{1}{2}-C \delta\right)$. If a Lieb-Thirring inequality (1.4) were to hold for $\gamma<1 / 2$ then for some finite $L_{\gamma}$

$$
c^{2 \gamma} \delta^{\gamma}\left(\frac{1}{2}-C \delta\right)^{\gamma} \leq \frac{L_{\gamma}}{b} \int_{\mathbb{R}} V(x)^{\gamma+\frac{1}{2}} \mathrm{~d} x=L_{\gamma} c^{\gamma+\frac{1}{2}}
$$

which is clearly a contradiction if $c \rightarrow 0$. 


\section{Acknowledgements}

A. Laptev was partially supported by RSF grant 18-11-0032. L. Schimmer was supported by VR grant 2017-04736 at the Royal Swedish Academy of Sciences. The authors would like to thank the anonymous referees for their useful comments to improve the article.

\section{References}

[1] Faddeev L.D., Takhtajan L.A., Liouville model on the lattice, in Field Theory, Quantum Gravity and Strings (Meudon/Paris, 1984/1985), Lecture Notes in Phys., Vol. 246, Springer, Berlin, 1986, 166-179.

[2] Frank R.L., Laptev A., Weidl T., Schrödinger operators: eigenvalues and Lieb-Thirring inequalities, in preparation.

[3] Gohberg I.C., Kreın M.G., Introduction to the theory of linear nonselfadjoint operators, Translations of Mathematical Monographs, Vol. 18, Amer. Math. Soc., Providence, R.I., 1969.

[4] Grassi A., Hatsuda Y., Mariño M., Topological strings from quantum mechanics, Ann. Henri Poincaré 17 (2016), 3177-3235, arXiv:1410.3382.

[5] Gu J., Klemm A., Mariño M., Reuter J., Exact solutions to quantum spectral curves by topological string theory, J. High Energy Phys. 2015 (2015), no. 10, 025, 69 pages, arXiv:1506.09176.

[6] Hundertmark D., Laptev A., Weidl T., New bounds on the Lieb-Thirring constants, Invent. Math. 140 (2000), 693-704, arXiv:math-ph/9906013.

[7] Hundertmark D., Lieb E.H., Thomas L.E., A sharp bound for an eigenvalue moment of the one-dimensional Schrödinger operator, Adv. Theor. Math. Phys. 2 (1998), 719-731, arXiv:math-ph/9806012.

[8] Hundertmark D., Simon B., Lieb-Thirring inequalities for Jacobi matrices, J. Approx. Theory 118 (2002), 106-130, arXiv:math-ph/0112027.

[9] Kashaev R., The quantum dilogarithm and Dehn twists in quantum Teichmüller theory, in Integrable Structures of Exactly Solvable Two-Dimensional Models of Quantum Field Theory (Kiev, 2000), NATO Sci. Ser. II Math. Phys. Chem., Vol. 35, Kluwer Acad. Publ., Dordrecht, 2001, 211-221.

[10] Kashaev R., Mariño M., Operators from mirror curves and the quantum dilogarithm, Comm. Math. Phys. 346 (2016), 967-994, arXiv:1501.01014.

[11] Kashaev R., Mariño M., Zakany S., Matrix models from operators and topological strings, 2, Ann. Henri Poincaré 17 (2016), 2741-2781, arXiv:1505.02243.

[12] Laptev A., Loss M., Schimmer L., On a conjecture by Hundertmark and Simon, arXiv:2012.13793.

[13] Laptev A., Schimmer L., Takhtajan L.A., Weyl type asymptotics and bounds for the eigenvalues of functional-difference operators for mirror curves, Geom. Funct. Anal. 26 (2016), 288-305, arXiv:1510.00045.

[14] Laptev A., Schimmer L., Takhtajan L.A., Weyl asymptotics for perturbed functional difference operators, J. Math. Phys. 60 (2019), 103505, 10 pages.

[15] Lieb E.H., Thirring W.E., Inequalities for the moments of the eigenvalues of the Schrödinger Hamiltonian and their relation to Sobolev inequalities, in Studies in Mathematical Physics, Princeton University Press, Princeton, 1976, 269-303.

[16] Takhtajan L.A., Trace formulas for the modified Mathieu equation, in Partial Differential Equations, Spectral Theory, and Mathematical Physics, Editors P. Exner, R. Frank, F. Gesztesy, H. Holden, T. Weidl, European Mathematical Society, Berlin, 2021, 427-443, arXiv:2103.00038.

[17] Takhtajan L.A., Faddeev L.D., The spectral theory of a functional-difference operator in conformal field theory, Izv. Math. 79 (2015), 388-410, arXiv:1408.0307. 